Meta

Journal des tradlucteurs

Translators' Journal

\title{
Lexicographe, terminologue, traducteur [trois mousquetaires à cheval]
}

\section{Henri Dumas}

Volume 19, numéro 3, septembre 1974

URI : https://id.erudit.org/iderudit/002674ar

DOI : https://doi.org/10.7202/002674ar

Aller au sommaire du numéro

Éditeur(s)

Les Presses de l'Université de Montréal

ISSN

0026-0452 (imprimé)

1492-1421 (numérique)

Découvrir la revue

Citer cet article

Dumas, H. (1974). Lexicographe, terminologue, traducteur [trois mousquetaires à cheval]. Meta, 19(3), 129-133. https://doi.org/10.7202/002674ar d'utilisation que vous pouvez consulter en ligne.

https://apropos.erudit.org/fr/usagers/politique-dutilisation/ 


\section{Lexicographe, terminologue, traducteur [trois mousquetaires à cheval]}

Nous avons pu lire dans un numéro récent de l'Actualité terminologique ${ }^{1}$, que le terminologue, Dieu merci, se portait bien, encore qu'il n'ait jamais été parrainé ni reconnu par son cousin le lexicographe. Usurpant sans vergogne les prérogatives de celui-ci, un terminologue avait même proposé pour celui-là la définition : lexicographe au service des traducteurs. Pourquoi, direz-vous, ne pas faire l'économie, toujours appréciable, d'un mot, en qualifiant l'un et l'autre de lexicographe? Parce que cette définition, pour être lapidaire, était trop simple, voire simpliste. En fait, lexicographe et terminologue, s'ils sont tous les deux au service de la même noble cause, la compréhension entre les hommes par la parole, procèdent de façon radicalement différente. Comme on dit chez les pétroliers : «Ils sont aux deux bouts du tuyau.»

Que fait le lexicographe? Nous vous invitons à relire les Actes du deuxième colloque international de linguistique et de traduction ${ }^{2}$, en particulier les articles de Bernard Quemada * Bilan des applications de l'informatique aux études lexicologiques » et d'Antonio Zampolli «L'automatisation de la recherche lexicologique ». Paraphrasant, à une majuscule près, l'Évangile, nous constatons qu'«au commencement était le verbe ». Les gens ont commencé à parler. Il faut croire qu'ils se sont compris, puisqu'ils ont continué. Ce n'est que tout récemment que certains ont pris le travers de parler pour ne rien dire ou de parler sans souci de se faire comprendre. Comment nos ancêtres cavernicoles pouvaient-ils se comprendre? C'est là une des premières merveilles du monde, mais le fait est qu'ils se comprenaient. Cependant, au fur et à mesure que se déliaient concomitamment esprits et langues, les notions, et les mots pour les exprimer, se multipliaient et la confusion serait aujourd'hui inextricable si des esprits éclairés n'avaient entrepris d'étudier systématiquement et scientifiquement les mots du langage. Ces esprits éclairés sont les lexicologues et les lexicographes. Rien ne saurait faire mieux comprendre

1. "Comment peut-on être terminologue?", l'Actualité terminologique, vol. 6, no 6 , juinjuillet 1973

2. Meta, vol. $18, \mathrm{n}^{\circ} 1-2$, mars-juin 1973 . 
le rôle du lexicographe, la nature de son travail, que la lecture de l'article de Zampolli que nous citions plus haut. Le lexicographe repère les mots, les recense, étudie les contextes qui les entourent, essaye de débrouiller et de comprendre les relations que symbolisent ces contextes, compare enfin toutes les relations ainsi identifiées pour essayer de trouver le dénominateur commun qui les satisfasse toutes, c'est-à-dire la notion commune, l'idée commune que les auteurs de ces contextes ont dû associer à chaque mot. Ce faisant, le lexicographe découvre le sens du mot, il ouvre l'enveloppe qui contenait le message, lit ce message, et le mot, compris et utilisable sans danger de méprise, devient terme. Il ne reste plus au lexicographe qu'à nous faire part de sa découverte, en nous donnant un résumé du message, une définition claire, précise, concise et élégante.

Le lexicographe part donc du mot pour arriver au sens.

Dans \& le cheval blanc d'Henri IV », que signifie le mot cheval? Jusqu'ici, tout ce que nous savons, c'est qu'il s'agit d'une chose, d'un être animé ou d'une personne susceptible d'être possédé par quelqu'un ou d'être en rapport avec quelqu'un. «Il est monté sur son cheval »: «son» confirme ce qui précède et «est monté » indique que le cheval est au-dessus du niveau du sol. "Une course de chevaux $»:$ il s'agit donc d'un animal, d'une personne ou d'un engin mécanique. «Mon cheval a mangé une botte de foin »: c'est donc un animal herbivore. «II a fait mettre quatre fers neufs aux sabots de son cheval»: cet animal est un ongulé, donc un mammifère. «Son cheval est bien dressé, cependant elle a voulu lui faire sauter une haie trop haute et sa monture est tombée les quatre fers en l'air »: animal domestique, quadrupède. Nous avons maintenant une idée suffisamment précise, grâce à l'analyse de ces quelques contextes, de ce que le mot «cheval» veut dire. Le lexicographe (du Petit Larousse) nous en donne un résumé, qu'il appelle définition :

Cheval. Mammifère domestique de l'ordre des ongulés, famille des équidés, remarquablement adapté à la course, caractérisé par l'allongement des membres et la possession d'un seul doigt à chaque patte.

Le terminologue, nous l'avons déjà dit, est «à l'autre bout du tuyau ». On peut lui demander, par exemple, quel est le terme français correspondant au mot anglais horse dans cette phrase à traduire : "The horse of Henri IV was white. 》 Notre terminologue n'a d'autre ressource que de consulter un lexicographe, celui du Webster en l'occurrence, qui lui tient exactement ce langage :

Horse : a large solid-hoofed herbivorous mammal domesticated by man since a prehistoric period and used as a beast of burden, a draft animal, or for riding, and distinguished from the other existing members of the genus Equus and family Equidae by the long hair of the mane and tail...

Il lui faut ensuite s'adresser à un autre lexicographe (celui de Larousse par exemple) pour lui demander, parmi tous les termes de la langue française dont il a déchiffré le sens et auxquels il a donné une définition, quel est celui dont le sens est le même que ce que nous propose le lexicographe anglais (ou américain) pour horse. En principe, il lui faudrait à chaque fois lire le Petit Larousse de A à $\mathrm{Z}$, jusqu'à ce qu'il trouve le terme désiré. Pour horse, il devra lire jusqu’à 
"cheval » à la page 198, mais pour assagai, il lui faudra aller jusqu'à «zagaie », à la page 1089. S'il ne trouve pas son bonheur. dans le Petit Larousse, il devra lire le Grand Larousse encyclopédique en 11 volumes, et, s'il ne l'y trouve pas, le Trésor de la langue française. En fait ce travail a, en général, déjà été fait et le résultat consigné dans des dictionnaires bilingues. Souvent le terminologue, ou le traducteur, ou le rédacteur, sait déjà quel terme convient probablement, sans être absolument sûr de sa signification exacte. Il lui suffit alors de chercher ce mot directement dans un dictionnaire.

Nous retenons donc ceci : alors que le lexicographe part du mot pour en trouver la signification, le terminologue part d'une signification et doit chercher un mot pour l'exprimer. Autre constatation : le lexicographe est au service de la langue et la rend intelligible, le terminologue est au service des usagers de la langue (rédacteurs ou traducteurs) et leur permet de s'exprimer et de se faire comprendre.

Existe-t-il une frontière infranchissable, un mur de Chine, un rideau de fer, entre le monde des lexicographes et celui des terminologues? Non.

Le lexicographe fait nécessairement œuvre de terminologue. Dès l'instant qu'il nous dit qu'il a compris que « cheval » désigne un mammifère domestique de l'ordre des ongulés, famille des équidés..., il nous suggère que si nous avons à parler d'un mammifère domestique de l'ordre des ongulés, famille des équidés, le terme qu'il convient d'employer est «cheval». En effet, tous ceux qui jusqu'à présent ont parlé de «cheval » entendaient par là ce que nous avons à exprimer, et ceux qui n'ont jamais entendu, vu ou utilisé ce mot, ni ne savent ce qu'il désigne, le lisant sous notre plume, consulterons le Petit Larousse, qui leur indiquera ce que l'on entend, ce que nous entendons par «cheval ».

Le terminologue, de son côté, fait parfois œuvre de lexicographe. En effet, toutes les notions, tous les objets qu'il doit nommer ne sont pas décrits même dans le dictionnaire le plus complet. La raison en est simple. Quand la pensée ou l'activité humaine (ce qui ne signifie pas que nous établissions une distinction fondamentale entre pensée et action) découvre une notion nouvelle ou invente un objet nouveau, le découvreur ou l'inventeur lui donne un nom; parfois même il le justifie et en offre une définition. Mais, avant que le mot n'apparaisse dans un dictionnaire, il faut qu'un lexicographe ait constaté : 1) son existence ; 2) son usage répété dans un certain nombre de contextes ; 3) que cet usage était justifié par l'absence de tout autre mot déjà reconnu et pouvant être utilisé à sa place ; 4) une signification commune dans les contextes relevés. De nos jours, le lexicographe dispose de moyens puissants (ordinateurs) pour effectuer ces tâches rapidement et avec un bon rendement. Mais cette productivité accrue ne fait que suivre la production toujours croissante de vocables nouveaux. Et puis, pour qu'il puisse constater l'usage répété du mot nouveau, il faut bien que quelqu'un l'ait utilisé, l'ait reconnu, en ait admis une définition. Ce quelqu'un, ce sont précisément des terminologues ou des rédacteurs ou des traducteurs, qui font de la lexicographie comme d'autres font de la prose, sans le savoir. 
Non, il n'y a pas de barrière infranchissable entre les domaines d'action du lexicographe et du terminologue. Qui plus est, on peut même dire qu'il existe un être aussi étrange et bizarre que le fabuleux centaure, moitié lexicographe et moitié terminologue : c'est le traducteur. Nous avons vu qu'une des premières tâches du lexicographe consistait à étudier des contextes et à essayer de débrouiller et de comprendre les relations que symbolisent ces contextes. Or, que fait le traducteur lorsqu'on lui présente un texte à traduire? Il le lit et essaye de le comprendre, il fait donc œuvre de lexicographe. Rares sont les termes qui désignent un seul concept ou objet strictement délimité. De toutes les acceptions possibles de chaque terme du texte à traduire, notre traducteur doit donc découvrir, en fonction du contexte, laquelle s'applique au cas présent : c'est un travail de lexicographe. Si un mot ne se trouve dans aucun dictionnaire de la langue de départ, le traducteur doit essayer de le retrouver dans d'autres contextes pour en découvrir le sens; s'il n'y réussit pas, il lui faudra se débrouiller avec un seul contexte. Si cette dernière entreprise est refusée par le lexicographe, car trop risquée, la première par contre, fait partie de ses tâches quotidiennes.

Ayant compris ou saisi le sens de chaque terme du texte original, le traducteur doit maintenant trouver les mots correspondants de la langue d'arrivée. Ceci est œuvre de terminologue, que le traducteur cherche et trouve ses mots lui-même, ce qui est préférable dans la mesure du possible, car c'est lui qui est le mieux à même d'apprécier la «situation» décrite en langue de départ; ou qu'il fasse appel à un terminologue patenté, spécialisé, qui fera la recherche ou le fera bénéficier des trouvailles d'autres traducteurs ayant agi en tant que terminologues.

Tire-t-on toujours profit de l'ambivalence des traducteurs? Il semble que non. Les résultats des recherches terminologiques que font les bons traducteurs ne sont pas exploités. Pour être exploités, il faudrait d'abord que ces résultats soient matérialisés, sous forme de fiches, puis recueillis, saisis, pour être ensuite analysés, critiqués, mis en forme et distribués. Or ceci ne se fait pas, malgré quelques vœux pieux à cet effet, qu'on trouve dans les exposés de fonctions des traducteurs et de leurs réviseurs.

L'activité de nature lexicographique des traducteurs n'est pas non plus exploitée, du moins à notre connaissance. Est-ce à dire qu'il faudrait demander au traducteur d'établir des fiches lexicologiques pour tous les mots non courants qu'il rencontre, y compris ceux qui n'ont pas posé de problème de traduction? Non. Le traducteur n'aurait plus le temps de traduire, une bonne partie de l'information ainsi captée serait inutile car redondante, et le circuit d'exploitation de cette information serait vite saturé. En outre, sì le traducteur établit des fiches terminologiques pour tous les vocables dont la traduction n'est pas évidente et immédiate, l'information lexicologique, limitée au contexte, peut trouver place sur ces fiches.

Cependant, il n'est pas interdit de rêver, voire d'entreprendre la recherche de faisabilité, d'un service de lexicographie attaché à un centre de traduction important. Ce service pourrait utiliser la capacité de traitement excédentaire du service de terminologie et s'alimenterait, dans leur version originale, de tous les textes traduits. Les dictionnaires à mise à jour continue, établis pour chacune 
des langues de départ, constitueraient, pour le service de terminologie et pour les traducteurs, des sources d'information fort utiles. Nous ne croyons pas que l'expérience ait jamais été tentée.

Sans aller si loin, il existe une autre méthode permettant de tirer le profit maximal du travail des traducteurs, en en récupérant la partie lexicographique. Elle consiste à faire fonctionner les traducteurs, de temps en temps, en circuit inversé (reverse flow). Le traducteur qui travaille toujours de l'anglais vers le français, par exemple, lit beaucoup d'anglais mais n'en tire pas profit, puisqu'il n'écrit jamais dans cette langue. Par contre, il n'a pas l'occasion, dans l'exercice de sa profession, de lire du français, donc d'enrichir sa terminologie, ce qui lui serait pourtant fort utile. Au contraire, le traducteur ambivalent, qui travaille alternativement dans les deux sens, s'enrichit de connaissances nouvelles. Il recharge, en quelque sorte, sa batterie (terminologique) chaque fois qu'il change de polarité.

Qu'ils soient réunis sous une même enveloppe de chair, ou qu'ils soient des individus distincts, lexicographe, terminologue et traducteur interviennent conjointement dans le travail de traduction. Pour que celui-ci soit « de la belle ouvrage » comme on dit chez nous, il faut bien sûr que le traducteur comprenne le texte qu'il doit faire comprendre à d'autres. Mais il est aussi essentiel que chacun des acteurs comprenne la vraie nature, le caractère fondamental du rôle qu'il joue dans l'acte de traduction. C'est pourquoi nous avons, par cet article, dirigé le spot sur chacun de nos trois acteurs, de nos trois mousquetaires, dont la devise est toujours : Tous pour un, un pour tous.

HenRI Dumas 